\title{
Packet Inter-Reception Time Modeling for High-Density Platooning in Varying Surrounding Traffic Density
}

\author{
Guillaume Jornod*†, Ahmad El Assaad*, Andreas Kwoczek* and Thomas Kürner ${ }^{\dagger}$ \\ ${ }^{*}$ Group Research, Volkswagen AG, Wolfsburg, Germany, \{guillaume.jornod,ahmad.el.assaad,andreas.kwoczek\}@ volkswagen.de \\ ${ }^{\dagger}$ Institut für Nachrichtentechnik, Technische Universität Braunschweig, Braunschweig, Germany, kuerner@ifn.ing.tu-bs.de
}

\begin{abstract}
A recent feature of communications systems is the agile quality of service adaptation, in which the application and the communications system exchange requirements and prediction of quality of service. The application first provides its quality of service requirement. The communications system tries to enforce it, and makes a prediction of the available quality of service. Finally, the application adapts its settings to the future quality of service and provides updated requirements. Though this concept is originally designed for cellular-based technologies, it is also applicable to ad-hoc communication systems.

In this paper, we focus on the prediction of quality of service for ad-hoc communications in a high-density platooning system. The quality of service of interest is the packet inter-reception time in an IEEE 802.11p network. Our platooning system drives through different vehicular traffic conditions, in which we gather transmission and position data. We then analyze the distribution of the packet inter-reception time to select the model features and then fit multiple distribution models. This empirical prediction modeling will then be the baseline for future modeling.
\end{abstract}

\section{INTRODUCTION}

Autonomous driving is on the rise. Though it has been demonstrated that autonomous vehicles can rely on their own sensors, it is foreseen that they can greatly benefit from cooperation, either in terms of control or in terms of perception. This cooperation is supported by vehicle-to-vehicle (V2V) or in general vehicle-to-everything (V2X) communications. When it comes to cooperative control, in safety-related time-critical applications, the quality of service (QoS) of communications system becomes an important feature in terms of functional safety. Indeed, the vehicular environment is very challenging for communications systems for its high variability. The main problem with V2X applications is the uncertainty in terms of QoS variation. An application that cannot predict the QoS cannot adapt its functioning to future condition and is therefore limited to the lower bound of its performance.

A novel concept in $\mathrm{V} 2 \mathrm{X}$ is agile quality of service adaptation (AQoSA) [1], in which the application and the network exchange QoS requirement and prediction respectively. The application uses the predictive quality of service (PQoS) to adapt its settings, such as the inter-vehicle distance (IVD) in a platooning systems. This concept can also be adapted to

This work has been supported in part by the Federal Ministry of Education and Research of the Federal Republic of Germany (BMBF) in the framework of the project 5G NetMobil with funding number 16KIS0681. The authors alone are responsible for the content of the paper. unassisted $\mathrm{V} 2 \mathrm{~V}$, where the on-board communications system itself provides the PQoS.

One interesting use case of AQoSA is high-density platooning (HDPL) supported by V2V, where targeted IVD are below $10 \mathrm{~m}$. In such configurations, the platooning system needs to exchange regular information with low end-to-end latency and high reliability. Particularly, the feasible IVD and the subsequent fuel efficiency will be dependent on the capability of the system in terms of reliability prediction.

If the packet error rate (PER) is an interesting measure of the channel QoS, it is even more relevant for the application to know what is the time between the reception of two messages from the same source, the packet inter-reception time (PIR), viz. how long it has to rely solely on its sensors and outdated information. While PER provides an information on the ratio transmission failures to number of transmissions, it does not reflect the pattern of these failures. This pattern is described by the PIR metric [2].

With a similar simulation environment as the one we use, [3] proposes to model PIR with exponential, Weibull, gamma and lognormal distributions. In this paper, we propose a datadriven method to derive this value from the environment. This method comprises surrounding environment modelling, features selection and distribution fitting. In the simulation results presented in this paper, we use a IEEE $802.11 \mathrm{p}$ radio access technology (RAT). The data-driven characteristic of our model allows a potential usage with any other V2X RATs. These encompass LTE-V, 5G-V2X and multi-RAT systems for instance.

Our environmental model aggregates the number of communicating vehicles in clusters in order to keep a constant number of features. We then apply kernel density estimation (KDE) for combinations of sub-intervals in the feature space to approach the probability density function (PDF) of the PIR. We show the results for selected dimensions from our feature space as well as from modified dimensions. The obtained results are promising for a first off-line learning approach, paving the way for further prediction models application and on-line training and evaluation.

The remaining of this paper is organized as following: Sec. II describes the scenario of interest and the simulation platform; Sec. III introduces the targets and the features of 
our model; Sec. IV presents the prediction model and selected results before Sec. V concludes this paper.

\section{SCEnARIo OF INTEREST}

In this section, we first describe the HDPL scenario. We then introduce the surrounding traffic that disturbs the functioning of the platoon. Finally, we present the simulation platform.

\section{A. High-Density Platooning}

A promising application of $\mathrm{V} 2 \mathrm{~V}$ is platooning. Vehicles in a platoon coordinate their manoeuvre to maintain constant IVD by using, for instance, cooperative adaptive cruise control (CACC). Doing so, they aim for increased safety and increased road efficiency. A particular case of platooning is HDPL, where these IVDs are below $10 \mathrm{~m}$. In addition to the objectives of platooning, HDPL also aims for fuel consumption reduction thanks to reduced air drag [4].

Our previous work highlights the challenges of $\mathrm{V} 2 \mathrm{~V}$ in platooning for different control strategies and manoeuvres [5], [6], [7]. In this paper, we aim to study the PIR for the maintain platoon manoeuvre, viz. with constant IVD and speed. As a result, the control strategy is not as important as when studying the impact on the application and is therefore not described in details. Our platooning system uses a CACC algorithm that is supported by the broadcast of platooning control messages (PCMs) transmitted to a maximum number of platoon members. The HDPL system is composed of five trucks of $18.8 \mathrm{~m}$ aiming for IVDs $\in\{0.2,0.5,1\} \mathrm{s}$ at $25 \mathrm{~m} / \mathrm{s}$, that is $5,12.5$ and $25 \mathrm{~m}$ respectively. PCMs have a size of $700 \mathrm{~B}$ and are sent at a rate of $20 \mathrm{~Hz}$ using IEEE 802.11p [8].

\section{B. Surrounding Traffic}

When the platoon is driving alone, the transmission of PCM is mostly altered by the pathloss, which is in our case mainly due to the distance between the transceivers. In an HDPL of five trucks of $18.8 \mathrm{~m}$ driving with $5 \mathrm{~m}$ (resp. 12.5 and $25 \mathrm{~m}$ ) headways, the maximum inter-antenna distance (IAD) is less than $100 \mathrm{~m}$ (resp. 130 and $180 \mathrm{~m}$ ), which stays within a reasonable communication range for the IEEE 802.11p RAT. Our aim is to study the impact of the spatial distribution of the surrounding traffic sending $400 \mathrm{~B}$ cooperative awareness message (CAM) [9] at $10 \mathrm{~Hz}$, which is the maximum rate allowed by the standard.

We therefore introduce over multiple simulation realizations more and more traffic on the three opposite lanes. We allow the incoming vehicles to drive with a maximum speed of $33 \mathrm{~m} / \mathrm{s}$ and to reach a minimum headway of $1 \mathrm{~m}$. This slightly unrealistic latter limitation lets us obtain extreme channel loads while keeping a realistic motion of the vehicles. These extreme cases are achieved with very high density of surrounding vehicles, as illustrated by Fig. 1 and Fig. 2(b). The number of vehicles within a range of $400 \mathrm{~m}$ around the transmitter reaches values over 200 vehicles.

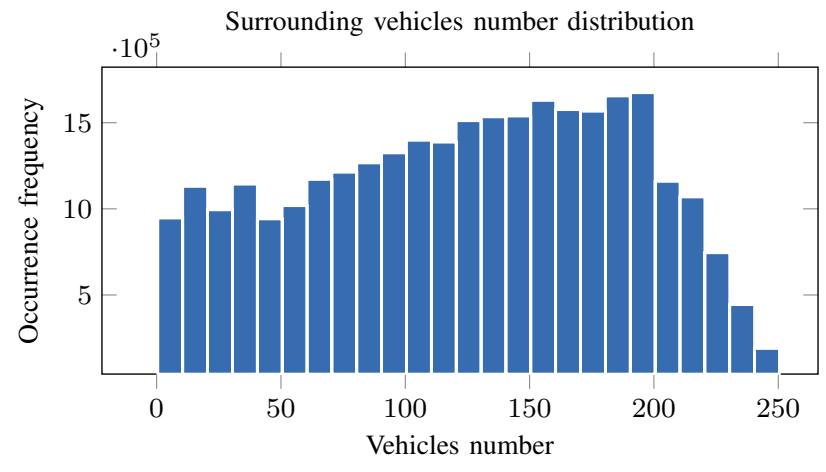

Fig. 1. Occurances of vehicles numbers within a range of $400 \mathrm{~m}$ from the transmitting vehicle along all simulations and timesteps.

\section{Simulation Platform}

Our platoon drives on a simulation replication of the test track Ehra-Lessien (see Fig. 2(a)). We use a combination of traffic and network simulators. We choose the former to be Simulation of Urban MObility (SUMO) [10] for its easy road network generation as well as for its capacity to spawn vehicles in a random fashion with different densities. It is interfaced with the network simulator, ns-3 [11], using the traffic control interface TraCI. The application layer creating the messages and the control strategy are both implemented in ns-3. We use the IEEE $802.11 \mathrm{p}$ implementation available in ns-3 [12] as communications system.

As a result, the vehicles mobility of the HDPL as well as the varying vehicular densities described in Secs. II-A and II-B are managed by SUMO, while the transmission of PCM and CAM are operated by ns-3. We use the tracing system available in ns-3 to log the reception of messages as well as the position of the nodes (platoon members as well as surrounding vehicles) at each timestep. In the scope of five experiments repeated three times for each IVD, we gather around $10^{6}$ transmission observations, along with the position of the nodes.

\section{TARGET AND FEATURES}

We present our model variables in this section. We first describe the variable that we try to predict and provide details on its computation. We then describe our environment abstraction and how it is processed to obtain the inputs for our prediction model.

\section{A. Target: Packet Inter-Reception Time}

Our QoS key performance indicator (KPI) is the PIR, $\gamma$, which is defined as the duration between two consecutive messages within a pair of communication partners, measured from the receiver. Formally, it is expressed as a function of $n_{\mathrm{L}}$, the consecutive dropped packets $(\mathrm{CDP}), T_{\mathrm{pcm}}=50 \mathrm{~ms}$, the PCM period, and $\tau$, some latency:

$$
\gamma=\left(n_{\mathrm{L}}+1\right) \cdot T_{\mathrm{pcm}}+\tau .
$$

Fig. 3 illustrates this relationship for one link and three packet drops. The latency is real $(\tau \in \mathbb{R})$ as it is the measured latency 


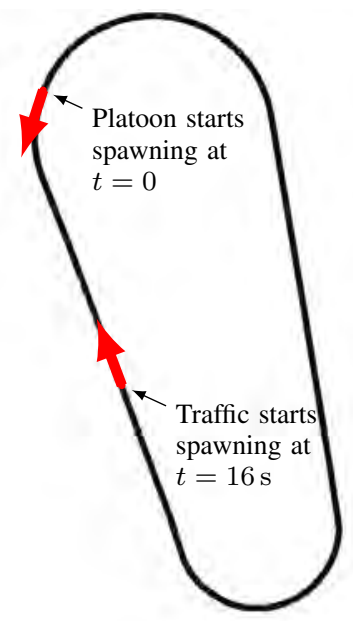

(a)

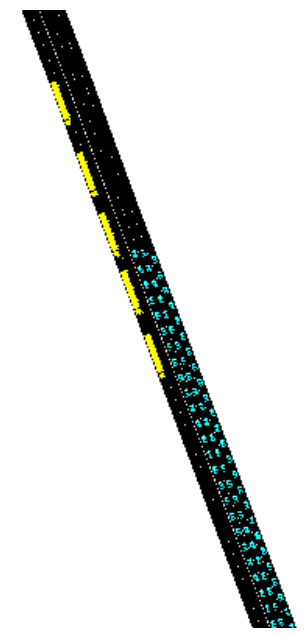

(b)
Fig. 2. Scenario snapshots: (a) overview of the Ehra-Lessien model with spawning positions times and driving directions (thick red arrows), and (b) zoom in on the five-truck platoon with extreme incoming traffic.

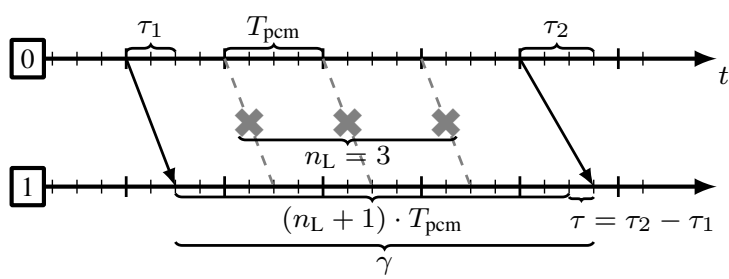

Fig. 3. PIR decomposition example for the link between node 0 and node 1 , with three consecutive packet drops $\left(n_{\mathrm{L}}=3\right)$. The latencies $\tau_{1}, \tau_{2}$ and $\tau$ are exaggerated for easier representation.

at the receiver, thus the difference between two latencies $\tau_{1}$ and $\tau_{2}$, the latencies of the two consecutive successful links. With $\tau_{1}>\tau_{2}$, the resulting latency $\tau$ is negative.

The prediction of PIR can also be reformulated as a discrete prediction problem, where CDP is a discrete variable. We then assume that $\tau$ will be at most $T_{\mathrm{pcm}}$, the transmission period of PCMs, and therefore can set $\tau=T_{\mathrm{pcm}}$ in Eq. (1) to have an upper-bound prediction of PIR:

$$
\begin{aligned}
\lceil\gamma\rceil & =\left(n_{\mathrm{L}}+1\right) \cdot T_{\mathrm{pcm}}+T_{\mathrm{pcm}} \\
& =\left(n_{\mathrm{L}}+2\right) \cdot T_{\mathrm{pcm}}
\end{aligned}
$$

Fig. 4 shows the distribution of the target as a continuous variable (PIR) and discrete variable (CDP). It highlights the challenge of the prediction, especially the high risk of overfitting in the low PIR region since the distribution is heavytailed, as also empahised in the experimental campaign presented in [2]. Indeed, even with dense surrounding — vehicular and communication - traffic creating a load on the channel, there is a significant chance of receiving the PCM with low PIR.

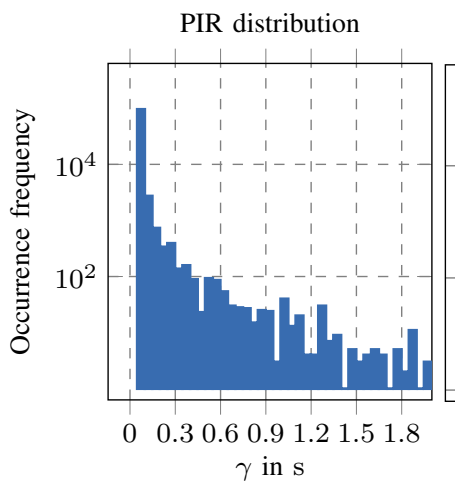

(a)
CDP distribution

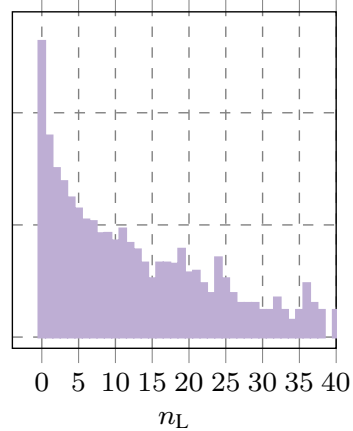

(b)
Fig. 4. Distribution of the prediction target in terms of (a) PIR and (b) CDP. The frequency is expressed in the logarithm form in order to facilitate the interpretation. This representation also highlights the heavy tail of the distribution of the variables.

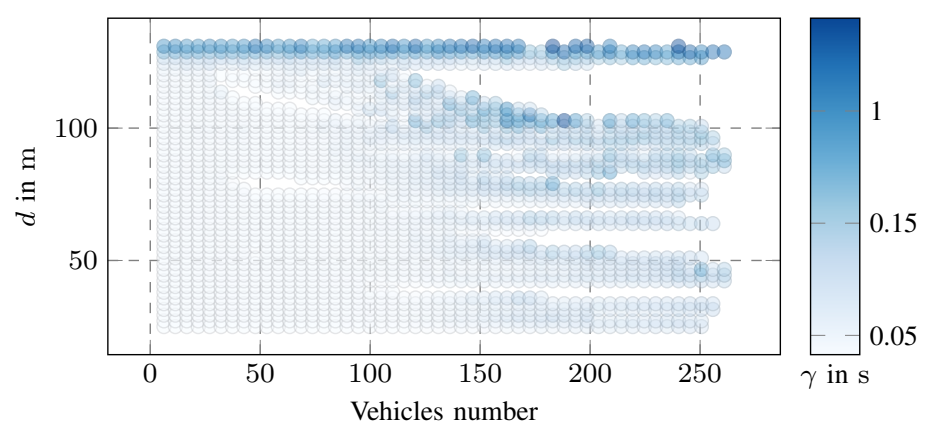

Fig. 5. Mean PIR as a function of the surrounding traffic within $400 \mathrm{~m}$ around the transmitter and the IAD. The mean is calculated from intersections of intervals of length 6 vehicles and $3 \mathrm{~m}$.

\section{B. Features Generation}

In the scope of a system-level simulation, two main factors have an impact on the communications performance: pathloss and channel load. The pathloss is mainly influenced by the distance between the transmitter and the receiver, and the channel is loaded by other ongoing communications. Fig. 5 shows the relationship between these two factors and the delayed reception of the messages. Small PIR occurs for low density and IAD, $d$, under $125 \mathrm{~m}$. Over this value, higher PIR times are appearing. These higher values are also more and more present for lower $d$ whilst the traffic densifies. Fig. 6 provides deeper insights on the distribution of PIR as a function of total number of vehicles within a radius of $400 \mathrm{~m}$ around the transmitter. Over 100 vehicles, the mean PIR starts to increase. Under approximately 175 vehicles, the third quartile is below the mean, showing the influence of the high values when $d>125 \mathrm{~m}$ shown in Fig. 5. These results corroborate our previous analysis of the PER for varying surrounding traffic densities and inter-transceiver distances [6].

Our goal is to create a model that represents the surrounding environment and to extract the QoS prediction in a technologyagnostic fashion. The main challenge is that most predic- 


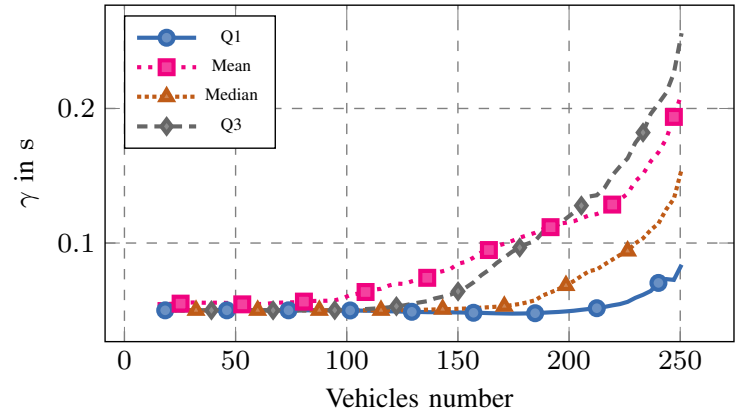

Fig. 6. Distribution of the PIR values as a function of the number of surrounding communicating vehicles. Q1 and Q3 denote the first and third quartiles.

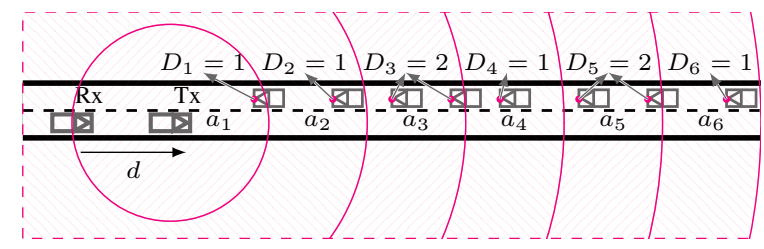

Fig. 7. Abstract representation of the environmental model features definition. $d$ is the IAD and $D_{n}$ is the number of surrounding vehicles in the annulus $a_{n}$.

tion models do not allow varying dimensionality of inputs, preventing to simply input the position of the surrounding vehicles. To workaround this issue, we aggregate the positions of the surrounding vehicles into an environment model. Fig. 7 illustrates this environment model, with $d$, the IAD, and $D_{n, g}$ the number of communicating vehicles (other than platoon members) within the areas $a_{n, g}$. The areas $a_{n, g}$ are concentric annuli of radius difference $g \in \mathbb{R}^{+}$centered around the transmitter, $n \in \mathbb{N}^{+}$being its index. In this illustrative example with arbitrary dimensions, $g$ is omitted. We count a vehicle in an annulus when its antenna is within the area. Formally, the annuli and the subsequent features are defined as:

$$
\begin{aligned}
a_{n, g} & =\left\{\boldsymbol{x} \in \mathcal{R} \mid(n-1) g \leq\left\|\boldsymbol{x}-\boldsymbol{x}_{\mathrm{t}}\right\|_{2}<n g\right\} \\
D_{n, g} & =\operatorname{card}\left(a_{n, g}\right),
\end{aligned}
$$

with $\mathcal{R}$ the set of the surrounding vehicle positions, $n$ the annulus index, $g$ its granularity value and $x_{\mathrm{t}}$ the position of the transmitter.

\section{Features Dimensionality}

In the following, the granularity parameter is set to $\mathrm{D}=$ $30 \mathrm{~m}$, and the largest considered range is $400 \mathrm{~m}$. Subsequently, the number of features is 14 (one distance and 13 areas). The number of surrounding vehicles has a larger negative influence on the performance of IEEE 802.11p for larger distances due to the hidden node problem in carrier sensing multiple access with collision avoidance (CSMA-CA) systems. It is therefore important to model the interactions between our features. To do so, we use the degree- 2 polynomial combinations of the features, considering distinct inputs only (ignoring $d^{2}$ for instance). As a result, the new feature space has $\sum_{i=1}^{2}\left(\begin{array}{c}14 \\ i\end{array}\right)=105$ dimensions.

A further step is to select the most influential and significant among these 105 features, to avoid increasing the complexity with insignificant or less impacting features. This step is performed using the results of scoring functions such as Ftest for PIR prediction. For our model, we select the 40 best features. These selected features are composed by the interactions between the distance and the six first areas as well as inter-areas interactions.

Alternatively, another dimensionality reduction method is principal component analysis (PCA). It has the advantage of creating a new space with uncorrelated variables, principal components. However, the influence of these new variables on our results is difficult to interpret as they do not have a physical meaning. In the following, we use the linear implementation provided by scikit-learn [13] with three components.

\section{Prediction Model and Results}

In this section, we formulate the prediction model and describe the method we use to approach the distribution of our target variables. Then, we present selected results and derive insights into the influence of our input parameters.

\section{A. Prediction Model Formulation}

Considering the high skewness of the distribution of PIR illustrated in Fig. 4, applying classical prediction may result in a systematic prediction of $\gamma=T_{\mathrm{pcm}}$ and $n_{\mathrm{L}}=0$ for regression and classification, respectively. Indeed, most estimator fitting algorithms reduce an error metric which is averaged over the observations, yielding potentially such results. To cope with this challenge, we propose to estimate the probability of the PIR or CDP values.

To do so, we split our dataset into intervals of equal length along each considered features (see Fig. 8 for a simplified example of feature space splitting). We then estimate the PDF for each of the subset. The length of the feature intervals may vary from one feature to another. As mentioned in the previous section, our model takes into account the 40 most influential and at the same time significant features. However, we can only represent three dimensions in our results section. In order to improve the interpretation potential we choose to graphically represent:

(i) Two large annuli- $D_{n, 200}$ with $n \in\{1,2\}$ - and the IAD;

(ii) The resulting three dimensions of a $\mathrm{PCA}-D_{n}^{\mathrm{P}}$ with $n \in$ $\{1,2,3\}$.

The produced model can then be used as a lookup table for the PIR probability. The data of the environmental model are gathered by the vehicle and transposed into the feature space (or using a PCA). The appropriate features are then matched to the intervals in the lookup table to finally obtain the PDF.

In the scope of an AQoSA system, the PDF is used to determine the occurrence probability of PIR values of interest. With this information on the reliability of the communications system, the platoon can assess the feasibility of decreasing 


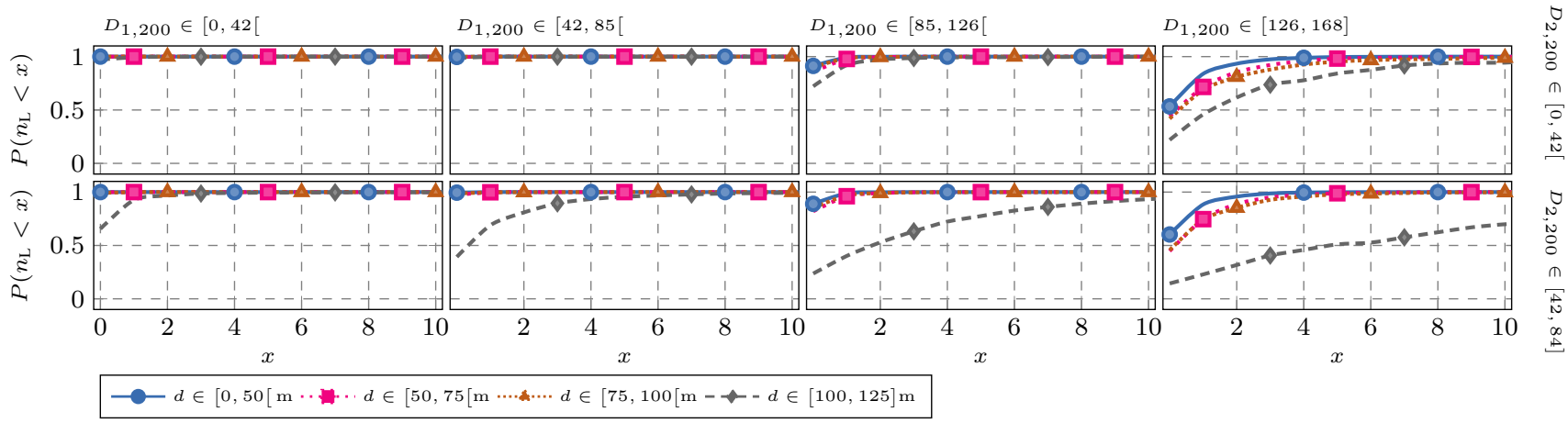

Fig. 8. CDFs resulting from CDP density estimation for physical dimensions. Each row represents the same vehicular density interval in the first annulus $D_{1}$, increasing from top to bottom. Each column represents the same vehicular density interval in the second annulus $D_{2}$, increasing from left to right. The curves represent different inter-antenna distance $d$ intervals. Too small datasets yield the absence of the corresponding curves.

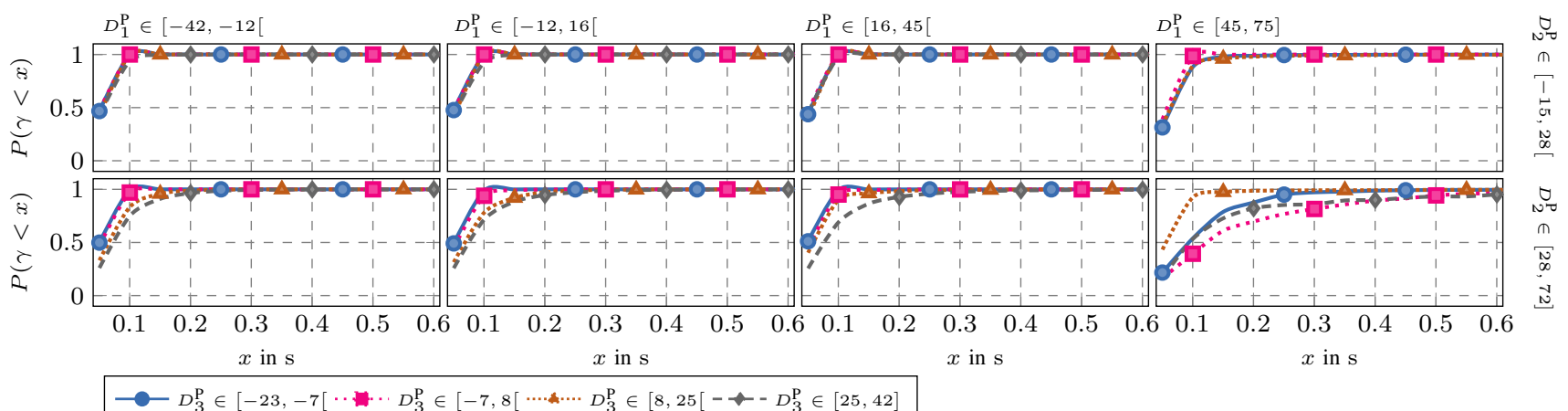

Fig. 9. CDFs resulting from PIR density estimation for PCA dimensions. Each row represents the same interval in the first dimension, increasing from top to bottom. Each column represents the same interval in the second dimension, increasing from left to right. The curves represent intervals of the third dimension. Too small datasets yield the absence of the corresponding curves.

the IVD. Indeed, the closer the vehicles, the higher the requirements on the communication systems.

\section{B. Kernel Density Estimation}

The PDF is estimated performing KDE, a non-parametric method commonly used for this purpose. The method has two hyper-parameters, the kernel and the smoothing bandwidth. The setting of these affects particularly the performances of the estimation. To select appropriately the two parameters, we systematically perform model selection using a grid search $k$ fold cross-validation (with $k=5$ ). The bandwidth is searched for between $10^{-2}$ and 10, whilst the kernel is chosen from the usual gaussian, exponential, tophat, Epenechnikov, linear and cosine kernels. Both KDE and model selection are using the python scikit-learn package [13]. If the bandwidth parameter varies along the whole search interval depending on the data subset being estimated, the selection results in exponential, a gaussian or a linear kernels.

\section{Selected Results}

The aforementioned high dimensionality issue prevents the representation of the whole model. Figs. 8 and 9 provide illustrative examples of the resulting estimated PDF for CDP and PIR, respectively, represented with their cumulative density functions (CDFs). The aim of these representations is to provide insights into the evolution of the distributions as a function of different parameters as well as to show the final product of the presented method, the prediction of PIR.

The full model has 40 dimensions, with the smallest intervals that the size of the dataset permits. The resulting dataset is saved in a database that is meant to be used in on-board systems as a lookup table for PIR prediction. Indeed, using gathered environment data, either with by creating the 40 features or the PCA variables, it is possible to check the appropriate intervals and obtain the density estimation for the target variable.

In Fig. 8, the highest probability to have more than 10 CDPs is found for the last intervals in terms of annuli vehicular density and distance. This is consistent with the intuition and the preliminary results previously presented. The trend is to have flatter CDFs when the traffic densifies and the transceivers get further away. By reducing the length of the intervals and reducing the area of the annuli, we can improve the granularity of the model. However, the risk is to have limited data sets that do not allow to perform KDE with sufficient significance.

Fig. 9 is more difficult to interpret as the dimensions do no represent real-world physical features. However, they yield a computationally faster PDF estimation, for they have fewer 


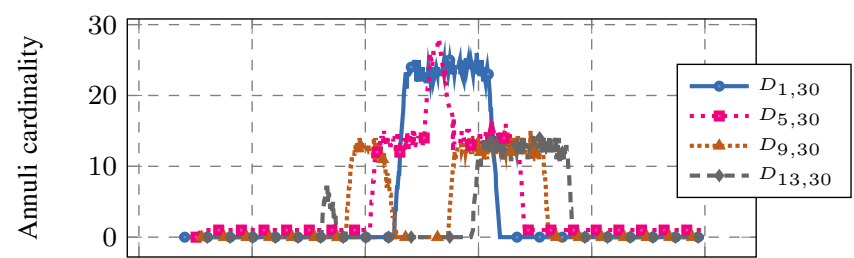

(a)

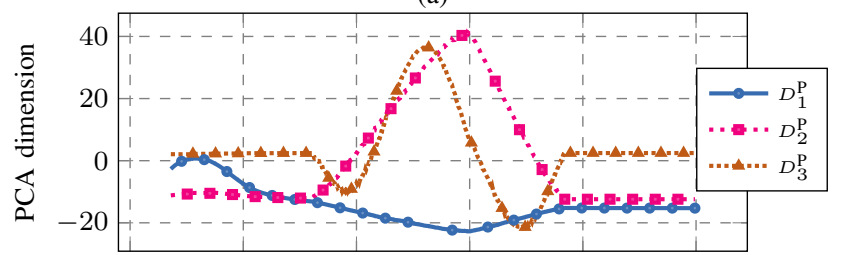

(b)

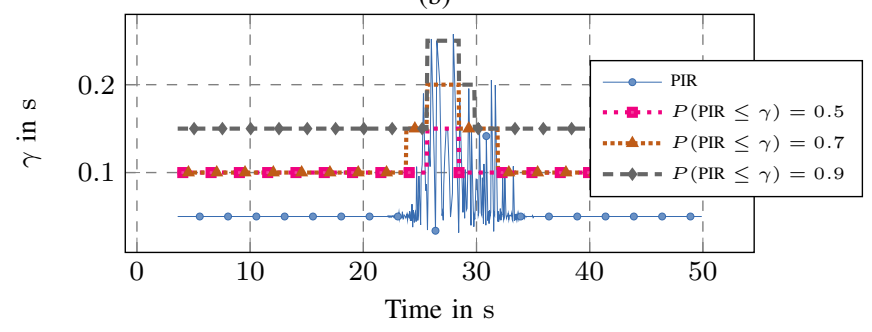

(c)

Fig. 10. On-line utilization example of the PCA-KDE model with 13 annul of size $g=30 \mathrm{~m}$ : (a) shows the number of vehicles in selected annuli; (b) provides the three resulting PCA features; (c) compares the observed PIR with the predicted values with different probabilities.

dimensions, requiring fewer models to be trained. We can also observe some signs of the input variables decorrelation, especially in the first figures row.

Fig. 10 illustrates the usage of the resulting look-up table for the prediction of PIR with PCA in one experiment. First, the polynomial combination of the 14 features, from which four are shown, are transformed into three principal components. At each timestep, the values of the three dimensions are looked for in the intervals presented in Fig. 9 to obtain the corresponding distribution. We can then compute the probability of the PIR time being under selected values. The figure shows the line of equal probabilities for three values, 0.5, 0.7 and 0.9. In this example, the model tends to be pessimistic for low surrounding traffic density. It successfully predicts the higher PIR values.

\section{CONCLUSION}

We presented a method for estimating the number of consecutive dropped packages and the packet inter-reception time. This method involves gathering transmission and position data, aggregating the data into our environmental model composed by geometrical features, and selecting the most significant resulting features. We then cut the resulting feature space into intervals and operate kernel density estimation. We show results for low dimensional cases in order to facilitate the representation and interpretation of the results. These results are then used in an on-line fashion to predict the PIR time for one link along one simulation experiment.

The channel model used in these experiments results is stochastic. One of the future step is to validate the transmission data against ray tracing data. The resulting density estimation will be the baseline for evaluating other statistical prediction models. Particularly, we aim at estimating this density continuously and on-line.

\section{REFERENCES}

[1] 5G Slicing Association, "5G Network Slicing for Cross Industry Digitization," 5G Slicing Association, Tech. Rep., 2018.

[2] M. E. Renda, G. Resta, P. Santi, F. Martelli, and A. Franchini, "IEEE 802.11 p VANets: Experimental evaluation of packet inter-reception time," Comput. Commun., vol. 75, pp. 26-38, 2016.

[3] M. Paul and G. Sanyal, "Traffic analysis of vehicular ad-hoc networks of V2I communication," Procedia Comput. Sci., vol. 54, pp. 215-223, 2015.

[4] A. Al Alam, A. Gattami, and K. H. Johansson, "An experimental study on the fuel reduction potential of heavy duty vehicle platooning," in 13th Int. IEEE Conf. Intell. Transp. Syst. (ITSC), 2010. IEEE, 2010, pp. 306-311.

[5] I. Llatser, G. Jornod, A. Festag, D. Mansolino, I. Navarro, and A. Martinoli, "Simulation of cooperative automated driving by bidirectional coupling of vehicle and network simulators," in IEEE Intell. Veh. Symp. (IV), 2017, pp. 1881-1886.

[6] G. Jornod, T. Nan, M. Schweins, A. El Assaad, A. Kwoczek, and T. Kürner, "Sidelink technologies comparison for highway high-density platoon emergency braking," in 2018 IEEE 16th Int. Conf. Intell. Transp. Syst. Telecommun. (ITST), Oct. 2018, pp. 1-7.

[7] G. Jornod, R. Alieiev, A. Kwoczek, and T. Kürner, "Environment-aware communications for cooperative collision avoidance applications," in 2018 IEEE 19th Int. Symp. "A World of Wireless, Mobile and Multimedia Networks" (WoWMoM), June 2018, pp. 588-599.

[8] "Intelligent Transport Systems (ITS); Performance evaluation of selforganizing TDMA as medium access control method applied to ITS; Access layer part," European Telecommunications Standards Institute, Tech. Rep. ETSI TR 102862 V1.1.1, 2011

[9] "Intelligent Transport Systems (ITS); Vehicular Communications; Basic Set of Applications; Part 2: Specification of Cooperative Awareness Basic Service ," Tech. Rep. ETSI EN 302 637-2, 2014.

[10] P. A. Lopez, M. Behrisch, L. Bieker-Walz, J. Erdmann, Y.-P. Flötteröd et al., "Microscopic traffic simulation using SUMO," in 21st IEEE Int. Conf. Intell. Transp. Syst. IEEE, 2018.

[11] G. F. Riley and T. R. Henderson, The ns-3 Network Simulator. Berlin, Heidelberg: Springer Berlin Heidelberg, 2010, pp. 15-34.

[12] J. Bu, G. Tan, N. Ding, M. Liu, and C. Son, "Implementation and evaluation of wave 1609.4/802.11 p in ns-3," in Proc. 2014 Workshop on $n s$-3. ACM, 2014, p. 1.

[13] F. Pedregosa, G. Varoquaux, A. Gramfort, V. Michel, B. Thirion et al., "Scikit-learn: Machine learning in Python," J. Mach. Learning Research, vol. 12, pp. 2825-2830, 2011. 